\title{
Prospects of discovering new physics in rare charm decays
}

\author{
Svjetlana Fajfer ${ }^{1,2, a}$, Nejc Košnik ${ }^{2, b}$ \\ ${ }^{1}$ Department of Physics, University of Ljubljana, Jadranska 19, 1000 Ljubljana, Slovenia \\ 2 J. Stefan Institute, Jamova 39, P. O. Box 3000, 1001 Ljubljana, Slovenia
}

Received: 28 October 2015 / Accepted: 19 November 2015 / Published online: 30 November 2015

(c) The Author(s) 2015. This article is published with open access at Springerlink.com

\begin{abstract}
The LHCb bounds on the branching ratio of the rare decay $D^{0} \rightarrow \mu^{+} \mu^{-}$and the constraints on the branching ratio of $D^{+} \rightarrow \pi^{+} \mu^{+} \mu^{-}$in the nonresonant regions enable us to improve constraints on new physics contributions. Using the effective Lagrangian approach we determine the sizes of the Wilson coefficients allowed by the existing LHCb bounds on rare charm decays. Then we discuss contributions to rare charm meson decay observables in several models of new physics: a model with an additional spin-1 weak triplet, leptoquark models, Two Higgs doublets model of type III, and a $Z^{\prime}$ model. Here we complement the discussion by $D^{0}-\bar{D}^{0}$ oscillations data. Among the considered models, only leptoquarks can significantly modify the Wilson coefficients. Assuming that the differential decay width for $D^{+} \rightarrow \pi^{+} \mu^{+} \mu^{-}$receives a NP contribution, while the differential decay width for $D^{+} \rightarrow \pi^{+} e^{+} e^{-}$is Standard Model-like, we find that lepton flavor universality can be violated and might be observed at high dilepton invariant mass.
\end{abstract}

\section{Introduction}

Processes with charmed mesons and top quarks offer an excellent opportunity to search for new physics (NP) in the up-type quark sector. In contrast to B meson physics, which is convenient to search for NP due to good exposure of the short-distance effects, charm quark systems are dominated by large long-distance quantum chromodynamics contributions. Such effects then screen the short-distance contributions of interest. Within the Standard Model (SM) the shortdistance physics in rare charm decays is strongly affected by the Glashow-Iliopoulos-Maiani (GIM) mechanism [1]. Namely, box or penguin diagram amplitudes get contributions from down-type quarks which are approximately massless from the weak scale perspective, and this warrants a very

\footnotetext{
${ }^{\text {a }}$ e-mail: svjetlana.fajfer@ijs.si

b e-mail: nejc.kosnik@ijs.si
}

effective GIM cancellation. Flavor changing neutral current (FCNC) processes with charm mesons might change charm quantum number for two or one unit $(|\Delta C|=2$ or $|\Delta C|=1$ transitions). The $|\Delta C|=2$ transition occurs in $D^{0}-\bar{D}^{0}$ oscillations and leads to strong constraints on NP from the measured observables as pointed out in [2,3]. There are two possibilities for NP in the $|\Delta C|=2$ transition: the transition might occur at tree level, in which case a new neutral scalar or a vector boson possesses FCNC couplings to $u$ and $c$ quarks, or at loop level via NP degrees of freedom affecting the box diagrams. The processes with $|\Delta C|=1$ on the quark level are $c \rightarrow u \gamma$ and $c \rightarrow u \ell^{+} \ell^{-}$[4-9]. Both transitions can be approached in the familiar effective Lagrangian formalism [3]. Additional constraints on NP arise from the down-type quark sector whenever new bosons couple to lefthanded quark doublets $[10,11]$. Since NP is very constrained by the current experimental results coming from $B$ and $K$ physics [12] the only chance to observe NP in rare charm decays seems to be when new bosons are coupled to weak singlets. This then allows one to avoid the strong flavor constraints in the down-type quark sector.

On the experimental side the LHCb experiment succeeded to improve the bound on the rates of $|\Delta C|=1$ decays by almost two orders of magnitude with respect to previous bounds. For the dileptonic decay the best bound to date is [13]

$\mathrm{BR}\left(D^{0} \rightarrow \mu^{+} \mu^{-}\right)<7.6 \times 10^{-9}$.

The above limit as well as other quoted limits in the following, unless stated otherwise, correspond to the $95 \% \mathrm{CL}$ upper bounds. In the decay $D^{+} \rightarrow \pi^{+} \mu^{+} \mu^{-}$the $\mathrm{LHCb}$ experiment focused on kinematic regions of dilepton mass, $q^{2}=\left(k_{-}+k_{+}\right)^{2}$, that are below or above the dominant resonant contributions due to vector resonances in the range $m_{\rho}^{2} \lesssim q^{2} \lesssim m_{\phi}^{2}$. The measured total branching ratio, obtained by extrapolating spectra over the resonant region, is [14]

$\operatorname{BR}\left(D^{+} \rightarrow \pi^{+} \mu^{+} \mu^{-}\right)<8.3 \times 10^{-8}$, 
while separate branching fractions in the low- and high- $q^{2}$ bins were bounded as $[14]^{1}$

$$
\begin{aligned}
& \operatorname{BR}\left(\pi^{+} \mu^{+} \mu^{-}\right)_{\mathrm{I}} \\
& \quad \equiv \mathrm{BR}\left(D^{+} \rightarrow \pi^{+} \mu^{+} \mu^{-}\right)_{q^{2} \in[0.0625,0.276] \mathrm{GeV}^{2}<2.5 \times 10^{-8}} \\
& \operatorname{BR}\left(\pi^{+} \mu^{+} \mu^{-}\right)_{\mathrm{II}} \\
& \equiv \mathrm{BR}\left(D^{+} \rightarrow \pi^{+} \mu^{+} \mu^{-}\right)_{q^{2} \in[1.56,4.00] \mathrm{GeV}^{2}<2.9 \times 10^{-8}}
\end{aligned}
$$

Motivated by these improved bounds we consider several NP models and either derive constraints on their flavor parameters and masses, or for the models that are severely bounded from alternative flavor observables (e.g. $D^{0}-\bar{D}^{0}$ mixing, $K$, or $B$ physics), we comment on the prospects of observing their signals in rare charm decays. To this end, we use the effective Lagrangian encoding the short-distance NP contributions in a most general way. Namely, the experimental results (1) and (3) give us a possibility to constrain NP in $c \rightarrow u \ell^{+} \ell^{-}$also in a model independent way.

In the case of $b \rightarrow s \ell^{+} \ell^{-}$transitions, LHCb has recently observed a large departure of the experimentally determined lepton flavor universality (LFU) ratio $R_{K}=\mathrm{BR}\left(B \rightarrow K \mu^{+} \mu^{-}\right)_{q^{2} \in[1,6] \mathrm{GeV}^{2} / \mathrm{BR}\left(B \rightarrow K e^{+}\right.}$ $\left.e^{-}\right)_{q^{2} \in[1,6] \mathrm{GeV}^{2}}$ from the expected SM value [15]. This value was found to be $R_{K}^{\mathrm{LHCb}}=0.745_{-0.074}^{+0.090} \pm 0.036$, lower than the SM prediction $R_{K}^{S M}=1.0003 \pm 0.0001$ [16]. This surprising result of LHCb indicates possible violation of LFU in the $\mu-e$ sector. Due to the importance of this result, we investigate whether analogous tests in the $\mu-e$ LFU can be carried out in $c \rightarrow u \ell^{+} \ell^{-}$processes.

The outline of this article is as follows. In Sect. 2 we describe effective Lagrangian of $|\Delta C|=1$ transition and determine bounds on the Wilson coefficients coming from the experimental limits on $\operatorname{BR}\left(D^{+} \rightarrow \pi^{+} \mu^{+} \mu^{-}\right)$and $\operatorname{BR}\left(D^{0} \rightarrow \mu^{+} \mu^{-}\right)$. Section 3 contains an analysis in the context of specific theoretical models of new physics, contributing to the $c \rightarrow u \ell^{+} \ell^{-}$and related processes. Section 4 discusses lepton flavor universality violation. Finally, we summarize the results and present conclusions in Sect. 5 .

\section{Observables and model independent constraints}

\subsection{Effective Hamiltonian for $c \rightarrow u \ell^{+} \ell^{-}$}

The relevant effective Hamiltonian at scale $\mu_{c} \sim m_{c}$ is split into three contributions corresponding to diagrams with intermediate quarks $q=d, s, b[9,17]$

\footnotetext{
${ }^{1}$ Note that the high- $q^{2}$ bin quoted by the experiment extends beyond the maximal allowed $q_{\max }^{2}=\left(m_{D}-m_{\pi}\right)^{2}=2.99 \mathrm{GeV}^{2}$.
}

$\mathcal{H}_{\mathrm{eff}}=\lambda_{d} \mathcal{H}^{d}+\lambda_{s} \mathcal{H}^{s}+\lambda_{b} \mathcal{H}^{\text {peng }}$,

where each of them is weighted by an appropriate combination $\lambda_{q}=V_{u q} V_{c q}^{*}$ of Cabibbo-Kobayashi-Maskawa (CKM) matrix elements. Virtual contributions of states heavier than charm quark are by convention contained within

$\mathcal{H}^{\text {peng }}=-\frac{4 G_{F}}{\sqrt{2}} \sum_{i=3, \ldots, 10} C_{i} \mathcal{O}_{i}$

The operators appearing in the above Hamiltonian have thus enhanced sensitivity to new physics contributions:

$$
\begin{aligned}
& \mathcal{O}_{7}=\frac{e m_{c}}{(4 \pi)^{2}}\left(\bar{u} \sigma_{\mu \nu} P_{R} c\right) F^{\mu \nu}, \quad \mathcal{O}_{S}=\frac{e^{2}}{(4 \pi)^{2}}\left(\bar{u} P_{R} c\right)(\bar{\ell} \ell), \\
& \mathcal{O}_{9}=\frac{e^{2}}{(4 \pi)^{2}}\left(\bar{u} \gamma^{\mu} P_{L} c\right)\left(\bar{\ell} \gamma_{\mu} \ell\right), \quad \mathcal{O}_{P}=\frac{e^{2}}{(4 \pi)^{2}}\left(\bar{u} P_{R} c\right)\left(\bar{\ell} \gamma_{5} \ell\right), \\
& \mathcal{O}_{10}=\frac{e^{2}}{(4 \pi)^{2}}\left(\bar{u} \gamma^{\mu} P_{L} c\right)\left(\bar{\ell} \gamma_{\mu} \gamma_{5} \ell\right), \quad \mathcal{O}_{T}=\frac{e^{2}}{(4 \pi)^{2}}\left(\bar{u} \sigma_{\mu \nu} c\right)\left(\bar{\ell} \sigma^{\mu \nu} \ell\right), \\
& \mathcal{O}_{T 5}=\frac{e^{2}}{(4 \pi)^{2}}\left(\bar{u} \sigma_{\mu \nu} c\right)\left(\bar{\ell} \sigma^{\mu \nu} \gamma_{5} \ell\right) .
\end{aligned}
$$

The chiral projectors are defined as $P_{L, R}=\left(1 \mp \gamma_{5}\right) / 2$, $F_{\mu \nu}$ is the electromagnetic field strength tensor. For each of the operators $\mathcal{O}_{7,9,10, S, P}$ we introduce the corresponding counterpart $\mathcal{O}_{7,9,10, S, P}^{\prime}$ with opposite chiralities of quarks. Within the SM the Wilson coefficients $C_{i}$ result from the perturbative dynamics of the electroweak interactions and $\mathrm{QCD}$ renormalization. The latter effect determines the value of $C_{7}\left(m_{c}\right)$ by two-loop mixing with current-current operators and was found to be $V_{c b}^{*} V_{u b} C_{7}^{\mathrm{SM}}=V_{c s}^{*} V_{u s}(0.007+$ $0.020 i)(1 \pm 0.2)[4,8]$. On the other hand the value of the $C_{9}$ Wilson coefficient was found to be small after including renormalization group running effects, as shown in [7] and confirmed in [6], while $C_{10}$ is negligible in the SM [18].

\section{$2.2 D^{+} \rightarrow \pi^{+} \mu^{+} \mu^{-}$}

In order to analyze NP effects in $D^{+} \rightarrow \pi^{+} \mu^{+} \mu^{-}$one needs to evaluate the hadronic transition matrix elements of currents $\bar{u} \gamma_{\mu} P_{L, R} c$ and $\bar{u} \sigma^{\mu \nu} P_{L, R} c$. The standard parametrization expresses these matrix elements in terms of three form factors:

$$
\begin{aligned}
& \left\langle\pi(k)\left|\bar{u} \gamma^{\mu}\left(1 \pm \gamma_{5}\right) c\right| D(p)\right\rangle \\
& =f_{+}\left(q^{2}\right)\left[(p+k)^{\mu}-\frac{m_{D}^{2}-m_{\pi}^{2}}{q^{2}} q^{\mu}\right] \\
& \quad+f_{0}\left(q^{2}\right) \frac{m_{D}^{2}-m_{\pi}^{2}}{q^{2}} q^{\mu}, \\
& \left\langle\pi(k)\left|\bar{u} \sigma^{\mu \nu}\left(1 \pm \gamma_{5}\right) c\right| D(p)\right\rangle \\
& =i \frac{f_{T}\left(q^{2}\right)}{m_{D}+m_{\pi}}\left[(p+k)^{\mu} q^{v}-(p+k)^{v} q^{\mu} \pm i \epsilon^{\mu \nu \alpha \beta}(p+k)_{\alpha} q_{\beta}\right],
\end{aligned}
$$


where $q=p-k$ is the dilepton four-momentum. For the $f_{+, 0}\left(q^{2}\right)$ form factors we use the Bečirević-Kaidalov (BK) parametrization [19]:

$$
\begin{aligned}
f_{+}\left(q^{2}\right) & =\frac{f_{+}(0)}{(1-x)(1-a x)}, \quad x=q^{2} / m_{\text {pole }}^{2}, \\
f_{0}\left(q^{2}\right) & =\frac{f_{+}(0)}{1-\frac{1}{b} x}
\end{aligned}
$$

with the shape parameters $m_{\text {pole }}$ and $a$ determined by measurements of $D \rightarrow \pi \ell v$ decay spectra. We make an average of four experimental fits to the shape parameters, by taking as input the CLEO-c tagged [20] and untagged analysis [21], BES III [22], and Babar [23] results, all compiled by the HFAG [24]. The fitted shape parameters are $m_{\text {pole }}=1.90(8) \mathrm{GeV}$ and $a=0.28(14)$. For the normalization of the form factor we rely on the lattice result $f_{+}(0)=0.67(3)$ calculated by the HPQCD Collaboration [25]. The shape parameter $b=1.27(17)$ has also been extracted in lattice simulations [26]. For the tensor current form factor we rely on the fit of lattice data to BK shape as in [26]:

$f_{T}\left(q^{2}\right)=\frac{f_{T}(0)}{(1-x)\left(1-a_{T} x\right)}$,

where $x=q^{2} / m_{D^{*}}^{2}, f_{T}(0)=0.46(4)$, and $a_{T}=0.18(16)$. Based on the effective Hamiltonian (4), the most general expression for the short-distance amplitude can be written as [27]

$$
\begin{aligned}
& \mathcal{A}_{\mathrm{SD}}\left(D^{+}(p) \rightarrow \pi^{+}\left(p^{\prime}\right) \mu^{+}\left(k_{+}\right) \mu^{-}\left(k_{-}\right)\right) \\
& =\frac{i G_{F} \lambda_{b} \alpha}{\sqrt{2} \pi}\left[V \bar{u} \not p v+A \bar{u} \not p \gamma_{5} v+(S+T \cos \theta) \bar{u} v\right. \\
& \left.\quad+\left(P+T_{5} \cos \theta\right) \bar{u} \gamma_{5} v\right] .
\end{aligned}
$$

Here $\theta$ is defined as the angle between the three-momenta of $B$ and $\ell^{-}$in the rest frame of lepton pair whereas $V, A, S, P, T$, and $T_{5}$ are $q^{2}$-dependent functions expressed in terms of hadronic form factors and Wilson coefficients,

$$
\begin{aligned}
V= & \frac{2 m_{c} f_{T}\left(q^{2}\right)}{m_{D}+m_{\pi}}\left(C_{7}+C_{7}^{\prime}\right)+f_{+}\left(q^{2}\right)\left(C_{9}+C_{9}^{\prime}\right) \\
& +\frac{8 f_{T}\left(q^{2}\right) m_{\ell}}{m_{D}+m_{\pi}} C_{T}, \\
A= & f_{+}\left(q^{2}\right)\left(C_{10}+C_{10}^{\prime}\right), \\
S= & \frac{m_{D}^{2}-m_{\pi}^{2}}{2 m_{c}} f_{0}\left(q^{2}\right)\left(C_{S}+C_{S}^{\prime}\right), \\
P= & \frac{m_{D}^{2}-m_{\pi}^{2}}{2 m_{c}} f_{0}\left(q^{2}\right)\left(C_{P}+C_{P}^{\prime}\right)-m_{\ell} \\
& \times\left[f_{+}\left(q^{2}\right)-\frac{m_{D}^{2}-m_{\pi}^{2}}{q^{2}}\left(f_{0}\left(q^{2}\right)-f_{+}\left(q^{2}\right)\right)\right] \\
& \times\left(C_{10}+C_{10}^{\prime}\right),
\end{aligned}
$$

$$
\begin{aligned}
& T=\frac{2 f_{T}\left(q^{2}\right) \beta_{\ell} \lambda^{1 / 2}}{m_{D}+m_{\pi}} C_{T}, \\
& T_{5}=\frac{2 f_{T}\left(q^{2}\right) \beta_{\ell} \lambda^{1 / 2}}{m_{D}+m_{\pi}} C_{T 5} .
\end{aligned}
$$

We have employed a shorthand notation $\lambda=\lambda\left(m_{D}^{2}, m_{\pi}^{2}, q^{2}\right)$, where $\lambda(x, y, z)=(x+y+z)^{2}-4(x y+y z+z x)$, as well as $\beta_{\ell}=\beta_{\ell}\left(q^{2}\right)=\sqrt{1-4 m_{\ell}^{2} / q^{2}}$. The decay spectrum can be expressed in terms of $q^{2}$-dependent angular coefficients as

$$
\begin{aligned}
& \frac{\mathrm{d} \Gamma(D \rightarrow \pi \ell \ell)}{\mathrm{d} q^{2} \mathrm{~d} \cos \theta} \\
& =N \lambda^{1 / 2} \beta_{\ell}\left[a_{\ell}\left(q^{2}\right)+b_{\ell}\left(q^{2}\right) \cos \theta+c_{\ell}\left(q^{2}\right) \cos ^{2} \theta\right], \\
& \quad N=\frac{G_{F}^{2}\left|\lambda_{b}\right|^{2} \alpha^{2}}{(4 \pi)^{5} m_{D}^{3}},
\end{aligned}
$$

whereas the angular coefficients are

$$
\begin{aligned}
a_{\ell}\left(q^{2}\right)= & \frac{\lambda}{2}\left(|V|^{2}+|A|^{2}\right)+8 m_{\ell}^{2} m_{D}^{2}|A|^{2} \\
& +2 q^{2}\left[\beta_{\ell}^{2}|S|^{2}+|P|^{2}\right] \\
& +4 m_{\ell}\left(m_{D}^{2}-m_{\pi}^{2}+q^{2}\right) \operatorname{Re}\left[A P^{*}\right], \\
\frac{b_{\ell}\left(q^{2}\right)}{4}= & q^{2} \beta_{\ell}^{2} \operatorname{Re}\left[S T^{*}\right]+q^{2} \operatorname{Re}\left[P T_{5}^{*}\right] \\
& +m_{\ell}\left(m_{D}^{2}-m_{\pi}^{2}+q^{2}\right) \operatorname{Re}\left[A T_{5}^{*}\right] \\
& +m_{\ell} \lambda^{1 / 2} \beta_{\ell} \operatorname{Re}\left[V S^{*}\right], \\
c_{\ell}\left(q^{2}\right)= & -\frac{\lambda \beta_{\ell}^{2}}{2}\left(|V|^{2}+|A|^{2}\right)+2 q^{2}\left(\beta_{\ell}^{2}|T|^{2}+\left|T_{5}\right|^{2}\right) \\
& +4 m_{\ell} \beta_{\ell} \lambda^{1 / 2} \operatorname{Re}\left[V T^{*}\right] .
\end{aligned}
$$

The coefficients $a_{\ell}$ and $c_{\ell}$ enter then the $q^{2}$ distribution of branching ratio whereas $b_{\ell}$ is proportional to the forwardbackward asymmetry:

$$
\begin{aligned}
\frac{\mathrm{dBR}}{\mathrm{d} q^{2}}(D \rightarrow \pi \ell \ell) & =\tau_{D} 2 N \lambda^{1 / 2} \beta_{\ell}\left[a_{\ell}\left(q^{2}\right)+\frac{1}{3} c_{\ell}\left(q^{2}\right)\right], \\
A_{\mathrm{FB}}\left(q^{2}\right) & \equiv \frac{\left(\int_{0}^{1}-\int_{-1}^{0}\right) \mathrm{d} \cos \theta \frac{\mathrm{d} \Gamma(D \rightarrow \pi \ell \ell)}{\mathrm{d} q^{2} \mathrm{~d} \cos \theta}}{\mathrm{d} \Gamma(D \rightarrow \pi \ell \ell) / \mathrm{d} q^{2}} \\
& =\frac{b_{\ell}\left(q^{2}\right)}{a_{\ell}\left(q^{2}\right)+\frac{1}{3} c_{\ell}\left(q^{2}\right)} .
\end{aligned}
$$

Contributions of the vector resonances $\rho, \omega$, and $\phi$, decaying to $\mu^{+} \mu^{-}$, is due to the first two terms in the effective Hamiltonian (4) and electromagnetic interaction. Effects of vector resonances to the spectrum can be treated assuming naïve factorization by adding a $q^{2}$-dependent piece to $C_{9}$ that contains the vector current of leptons. Analogously, the scalar contribution of $\eta$ feeds into $C_{S}$. The procedure is described in detail in Ref. [28] for the contribution of $D^{+} \rightarrow \pi^{+} \rho^{0}(\omega)$ and updated for $D^{+} \rightarrow \pi^{+} \phi \rightarrow \pi^{+} \mu^{+} \mu^{-}$in Ref. [26]. The current experimental upper bound outside the resonance region indicates that the long-distance contribution is very 
suppressed. One might expect that at high invariant dilepton mass bin some excited states of vector mesons might give an additional long-distance contribution. However, it was shown in $[29,30]$ that contributions of these states are negligible in comparison with the leading long-distance contributions. We parametrize the resonances with the Breit-Wigner shapes,

$$
\begin{aligned}
C_{9}^{\mathrm{res}}= & \frac{\lambda_{d}}{\lambda_{b}}\left[a_{\rho} \frac{m_{\rho}^{2}}{q^{2}-m_{\rho}^{2}+i \sqrt{q^{2}} \Gamma_{\rho}}\right. \\
& \left.+a_{\omega} \frac{m_{\omega}^{2}}{q^{2}-m_{\omega}^{2}+i m_{\omega} \Gamma_{\omega}}-a_{\phi} \frac{m_{\phi}^{2}}{q^{2}-m_{\phi}^{2}+i m_{\phi} \Gamma_{\phi}}\right] \\
C_{S}^{\mathrm{res}}= & \frac{\lambda_{d}}{\lambda_{b}} \frac{a_{\eta} m_{\eta}^{2}}{q^{2}-m_{\eta}^{2}+i m_{\eta} \Gamma_{\eta}} .
\end{aligned}
$$

The magnitude of unknown parameters $a_{X}(X=\rho, \omega, \phi, \eta)$, can be fitted to the measured resonant branching ratios, given in Table 1 [31]. The corresponding values of $\left|a_{X}\right|$ are given in the second row in Table 1. We treat the relative phases as free parameters. Alternatively, for the relative phases and magnitudes of $a_{X}$ one can use flavor structure arguments [18]. In the left-hand panel in Fig. 1 we present the long-distance contributions to the differential branching ratio for $D^{+} \rightarrow \pi^{+} \mu^{+} \mu^{-}$as a function of dilepton invariant mass for a representative set of parameters $\left|a_{X}\right|$ from the $1 \sigma$ region (Table 1 ) and random phases of $a_{X}$. On the righthand panel in Fig. 1 we also indicate the interpretation of experimental upper bounds (3) in the case where the total amplitude would be constant, namely in the case where all angular coefficient functions $a_{\ell}, b_{\ell}, c_{\ell}$ would be independent of $q^{2}$. We also estimate the saturation of these bounds by the

Table $11 \sigma$ ranges and $90 \%$ CL upper bounds on resonant branching ratios and amplitude parameters [31]

\begin{tabular}{lllll}
\hline$X$ & $\rho$ & $\omega$ & $\phi$ & $\eta$ \\
\hline $\begin{array}{l}\mathrm{BR}\left(D^{+} \rightarrow \pi^{+} X(\rightarrow\right. \\
\left.\left.\mu^{+} \mu^{-}\right)\right)\left[10^{-8}\right]\end{array}$ & $3.7(7)$ & $<3.1$ & $160(10)$ & $2.0(3)$ \\
$\left|a_{X}\right|$ & $1.21(12)$ & $<0.26$ & $0.94(3)$ & $0.27(2)$ \\
\hline
\end{tabular}

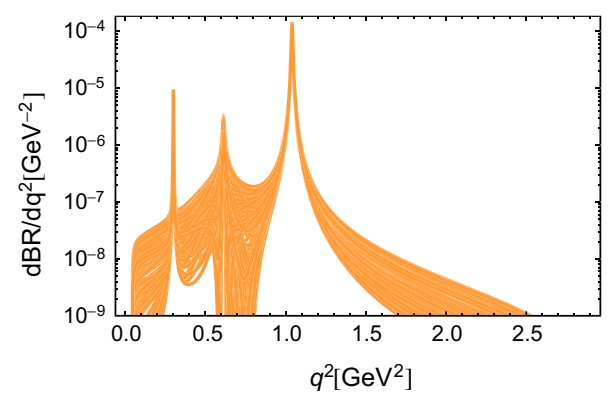

total resonant decay branching ratio and find for the lowand high- $q^{2}$ bin contributions to be smaller than $7.3 \times 10^{-9}$ and $5.3 \times 10^{-9}$, respectively. On the other hand, the shortdistance contribution to the total branching ratio of the SM due to the quoted value of $C_{7}$ is of the order $10^{-12}$ and thus negligible.

The branching ratio for $D^{0} \rightarrow \mu^{+} \mu^{-}$can be written in its most general form as

$$
\begin{aligned}
\operatorname{BR}( & \left.D^{0} \rightarrow \mu^{+} \mu^{-}\right) \\
= & \frac{1}{\Gamma_{D}} \frac{G_{F}^{2} \alpha^{2}}{64 \pi^{3}}\left|V_{c b}^{*} V_{u b}\right|^{2} f_{D}^{2} m_{D}^{3} \beta_{\mu}\left(m_{D}^{2}\right) \\
& \times\left[\left|\frac{2 m_{\mu}}{m_{D}}\left(C_{10}-C_{10}^{\prime}\right)+\frac{m_{D}}{m_{c}}\left(C_{P}-C_{P}^{\prime}\right)\right|^{2}\right. \\
& \left.+\frac{m_{D}^{2}}{m_{c}^{2}} \beta_{\mu}\left(m_{D}^{2}\right)^{2}\left|\left(C_{S}-C_{S}^{\prime}\right)\right|^{2}\right],
\end{aligned}
$$

where the decay constant of a $D$ meson, $f_{D}=209$ (3) MeV, has been averaged over $N_{f}=2+1$ lattice simulations [3234]. In the SM this decay is dominated by the intermediate $\gamma^{*} \gamma^{*}$ state that is electromagnetically converted to a $\mu^{+} \mu^{-}$ pair. It was estimated in [9] that $\operatorname{BR}\left(D^{0} \rightarrow \mu^{+} \mu^{-}\right) \simeq 2.7$. $10^{-5} \times \mathrm{BR}\left(D^{0} \rightarrow \gamma \gamma\right)$, and, together with the upper bound $\mathrm{BR}\left(D^{0} \rightarrow \gamma \gamma\right)<2.2 \times 10^{-6}$ at $90 \% \mathrm{CL}$ [35], this leads to the $\operatorname{limit} \operatorname{BR}\left(D^{0} \rightarrow \mu^{+} \mu^{-}\right)^{\mathrm{SM}} \lesssim 10^{-10}$.

\section{Constraints on the Wilson coefficients}

In this section we interpret the experimental bounds in kinematical regions I and II given in Eq. (3) as constraints on benchmark scenarios with NP contributions affecting individual Wilson coefficients. In the nonresonant regions of $D^{+} \rightarrow \pi^{+} \mu^{+} \mu^{-}$the long-distance resonant contributions are one order of magnitude below the current experimental sensitivity. This allows us to saturate experimental results for the differential decay width distribution at the low/high dilepton invariant mass bins by the contributions of the effective Wilson coefficients. In Fig. 2 we show the kinematical effect of setting to 1 individual Wilson coefficients one at

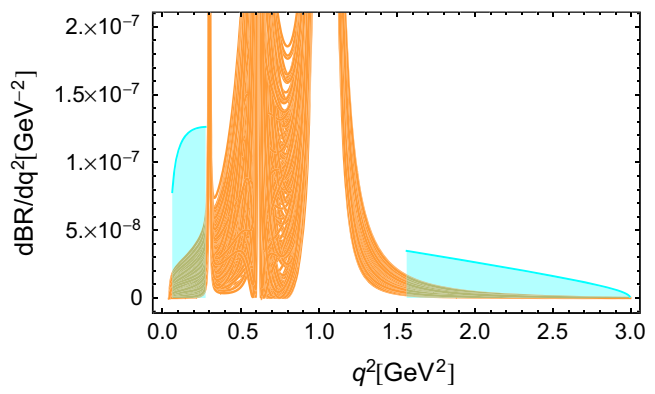

Fig. 1 SM resonant contributions in $D^{+} \rightarrow \pi^{+} \mu^{+} \mu^{-}$shown in orange. On the right-hand side panel cyan regions correspond to a scenario with constant decay amplitude that would saturate $\mathrm{LHCb}$ bounds (3) 


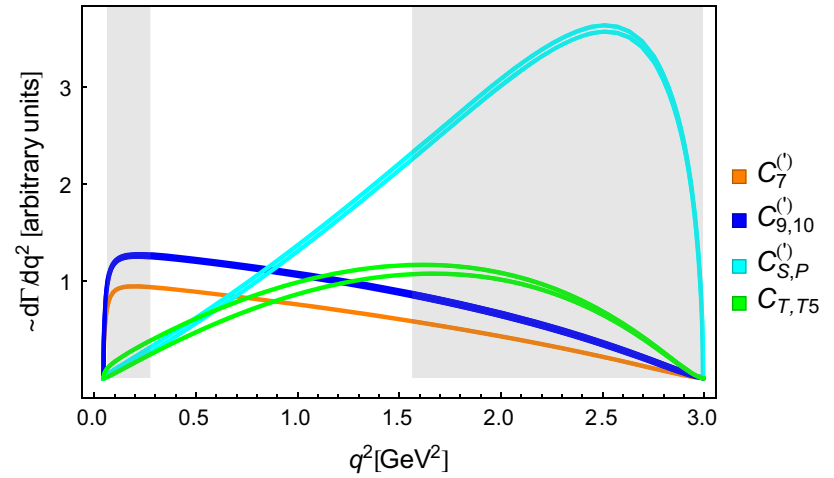

Fig. 2 Comparison of short-distance spectrum sensitivities to different Wilson coefficients. Grey regions indicate the LHCb experimental lowand high- $q^{2}$ bins

the time, where we have neglected the SM resonant contributions. A strong kinematical dependence of spectra in the cases of tensor and especially pseudoscalar coefficients suggests they will be better constrained in the high- than in the low- $q^{2}$ bin. For EM dipole and (axial-)vector interactions the enhancement at low- $q^{2}$ bin is hindered by relatively smaller phase space devoted to that bin.

We allow only one Wilson coefficient at a time to have a real nonzero value and extract its upper bound. This is repeated for each choice of random phases and moduli of the Breit-Wigner parameters $a_{\eta, \rho, \omega, \phi}$, where the latter are sampled in their $1 \sigma$ regions ( $90 \%$ CL bound for $\left|a_{\omega}\right|$ ); cf. Table 1 . The most relaxed bound obtained in this way is then reported in Table 2, where we use the notation $\tilde{C}_{i}=V_{u b} V_{c b}^{*} C_{i}$. At the same time the branching ratio of $D^{0} \rightarrow \mu^{+} \mu^{-}$can give bounds on the Wilson coefficients $C_{10}, C_{S}$, and $C_{P}$. It turns out that the upper bound on $\operatorname{BR}\left(D^{0} \rightarrow \mu^{+} \mu^{-}\right)$is more restrictive for the $C_{S, P, 10}$ Wilson coefficients than any of the invariant dilepton mass bins of $D^{+} \rightarrow \pi^{+} \mu^{+} \mu^{-}$.

The high invariant dilepton mass bin is more restrictive than the low dilepton invariant mass bin. Due to the parity conservation in $D \rightarrow \pi$ transition the bounds for $\tilde{C}_{j}^{\prime}, j=$ 7, 9, 10, $S, P$ are the same as for $\tilde{C}_{j}$.

In specific cases the angular distribution with respect to $\cos \theta$ can be a good discriminant between the resonant and genuine short-distance contributions. It was shown that the forward-backward asymmetry (FBA) can be enhanced toward the larger end of the $q^{2}$-spectrum in models with tensor and scalar Wilson coefficients (or pseudoscalar and pseudotensor) simultaneously present [36]. In principle such a scenario can be realized by a nonchiral leptoquark to be discussed in the following section. As a numerical example we choose $\tilde{C}_{S}=0.049$, allowed by the $D^{0} \rightarrow \mu^{-} \mu^{+}$, and in addition $\tilde{C}_{T}=0.2$, which results in $\operatorname{BR}\left(D^{+} \rightarrow\right.$ $\left.\pi^{+} \mu^{+} \mu^{-}\right)_{\text {II }}<10^{-8}$ and is therefore hard to distinguish from the resonant background. On the other hand, the FBA
Table 2 Maximal allowed values of the Wilson coefficient moduli, $\left|\tilde{C}_{i}\right|=\left|V_{u b} V_{c b}^{*} C_{i}\right|$, calculated in the nonresonant regions of $D^{+} \rightarrow \pi^{+} \mu^{+} \mu^{-}$in the low lepton invariant mass region $\left(q^{2} \in\right.$ $\left.[0.0625,0.276] \mathrm{GeV}^{2}\right)$, denoted by I, in the high invariant mass region $\left(q^{2} \in[1.56,4.00] \mathrm{GeV}^{2}\right)$, denoted by II, and from the upper bound $\mathrm{BR}\left(D^{0} \rightarrow \mu^{+} \mu^{-}\right)<7.6 \times 10^{-9}$ [13]. The last row gives the maximal value for the case where $\tilde{C}_{9}= \pm \tilde{C}_{10}$. All the quoted bounds have been derived for real $C_{i}$. The bounds for $\tilde{C}_{i}$ apply also to the chirally flipped coefficients $\tilde{C}_{j}^{\prime}$

\begin{tabular}{llll}
\hline & $\left|\tilde{C}_{i}\right|_{\max }$ & & \\
\cline { 2 - 4 } & $\mathrm{BR}(\pi \mu \mu)_{\mathrm{I}}$ & $\mathrm{BR}(\pi \mu \mu)_{\mathrm{II}}$ & $\mathrm{BR}\left(D^{0} \rightarrow \mu \mu\right)$ \\
\hline$\tilde{C}_{7}$ & 2.4 & 1.6 & - \\
$\tilde{C}_{9}$ & 2.1 & 1.3 & - \\
$\tilde{C}_{10}$ & 1.4 & 0.92 & 0.63 \\
$\tilde{C}_{S}$ & 4.5 & 0.38 & 0.049 \\
$\tilde{C}_{P}$ & 3.6 & 0.37 & 0.049 \\
$\tilde{C}_{T}$ & 4.1 & 0.76 & - \\
$\tilde{C}_{T 5}$ & 4.4 & 0.74 & - \\
$\tilde{C}_{9}= \pm \tilde{C}_{10}$ & 1.3 & 0.81 & 0.63 \\
\hline
\end{tabular}

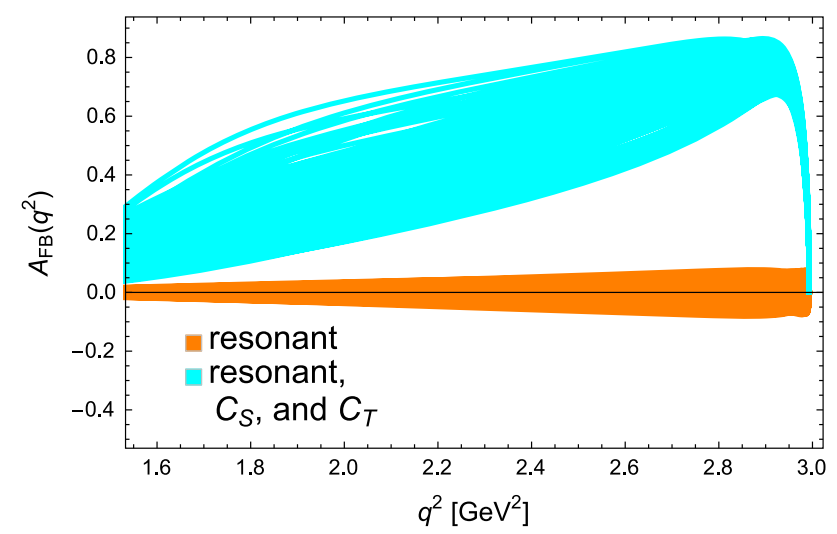

Fig. 3 Forward-backward asymmetry for the resonant background itself (orange) and in the scenario with $C_{S}=0.049 / \lambda_{b}, C_{T}=0.2 / \lambda_{b}$ (cyan)

in this case is strongly enhanced in the high- $q^{2}$ region, as shown in Fig. 3.

We turn to the discussion of specific models in the next section.

\section{Impact on specific models}

\subsection{Spin-1 weak triplet}

Introducing an additional vector particle that transforms as a triplet under $S U(2)_{L}$ affects a plethora of flavor observables. It has been shown recently that a model of this type explains the current $B$ sector anomalies $\left(R_{D^{(*)}}, R_{K}\right)$ even in the scenario with $U(2)_{q} \times U(2)_{\ell}$ flavor symmetry [11]. The 
relevant effective Lagrangian that follows from integrating out the vector triplet at tree level reads

$\mathcal{L}=-\frac{1}{2 m_{V}^{2}} J_{\mu}^{a} J^{a \mu}$,

representing a contact interaction between vector currents of left-handed quark and lepton doublets:

$J_{\mu}^{a}=g_{q} \lambda_{i j}^{q}\left(\bar{Q}^{i} \gamma_{\mu} T^{a} Q^{j}\right)+g_{\ell} \lambda_{i j}^{\ell}\left(\bar{L}^{i} \gamma_{\mu} T^{a} L^{j}\right)$.

The indices of $\lambda^{q}$ and $\lambda^{\ell}$ denote the mass-eigenstates of down-type quarks and charged leptons. The Hermitian matrices $\lambda_{i j}^{q, \ell}$ are conventionally normalized to $\lambda_{33}^{q}=\lambda_{33}^{\ell}=1$, with subleading entries in $\lambda_{3 i}$, whereas entries involving only the first two generations of quarks are severely suppressed. This hierarchy is a direct consequence of the imposed flavor symmetry. The dominant contributions to the processes involving only the first two generations are induced by $\lambda_{b b}^{q}$ and accompanying CKM rotations. Following [11] for the rare charm decays these are

$\mathcal{L}_{\mathrm{FCNC}}=\frac{g_{q} g_{\ell}}{4 m_{V}^{2}} \lambda_{a b}^{\ell}\left(V \lambda^{q} V^{\dagger}\right)_{i j}\left(\bar{\ell}^{a} \gamma_{\mu} P_{L} \ell^{b}\right)\left(\bar{u}^{i} \gamma_{\mu} P_{L} u^{j}\right)$,

where the quark sector couplings originate dominantly from the CKM mixing, namely $\left(V \lambda^{q} V^{\dagger}\right)_{u c} \approx V_{u b} V_{c b}{ }^{*}$. A purely left-handed current effective interaction generates the following pair of Wilson coefficients:

$$
-C_{10}=C_{9}=R_{0} \lambda_{\mu \mu}^{\ell} \frac{\pi}{\alpha}
$$

where $R_{0}=\left(g_{q} g_{\ell} m_{W}^{2}\right) /\left(g^{2} m_{V}^{2}\right)$ is directly related to the LFU $\tau / \ell$ ratio $R_{D^{(*)}}$ in semileptonic $B$ meson decays whose experimental value requires $R_{0}=0.14 \pm 0.04$. The constraint from $\tau \rightarrow 3 \mu$ implies $\lambda_{\mu \mu}^{\ell}=(0.013 \pm 0.011)\left(0.15 / R_{0}\right) \frac{g_{q}}{g_{\ell}}$, while the constraint on the $|\Delta C|=2$ operators from $C P$ violation in $D^{0}-\bar{D}^{0}$ mixing results in the inequality $g_{\ell} / g_{q}>$ $1.26 R_{0}$. From these ingredients one can estimate the maximum value of $C_{9}$,

$C_{9} \simeq 50 \frac{0.013 \pm 0.011}{R_{0}} \lesssim 10$,

which is unfortunately too small to have a detectable effect in $D \rightarrow \pi \mu^{+} \mu^{-}$or in $D^{0} \rightarrow \mu^{+} \mu^{-}$.

\subsection{Leptoquarks}

There exist several scalar and vector leptoquark (LQ) states which may leave imprint on $c \rightarrow u \ell^{+} \ell^{-}$transitions [37]. The possible scalar states transform under the SM gauge group as $(3,3,-1 / 3),(3,1,-1 / 3)$, and $(3,2,7 / 6)$, of which only the latter state conserves baryon and lepton number on the renormalizable level. Thus the mass of the scalar multiplet $(3,2,7 / 6)$ can be close to the electroweak scale without destabilizing the proton. In addition, there are four vector LQs which potentially contribute in rare charm decays, and they carry the following quantum numbers: $(3,3,2 / 3)$, $(3,1,5 / 3),(3,2,1 / 6)$, and $(3,2,-5 / 6)$. Only the first two states have definite baryon and lepton numbers.

Among all scalar LQs we will consider only the baryon number conserving state $(3,2,7 / 6)$, which comes with a rich set of couplings that are in general severely constrained by $B$ and $K$ physics [38]. Then, among the two baryon number conserving vector LQs we will focus on the state in the representation $(3,1,5 / 3)$ whose phenomenology is limited to the up-type quarks and charged leptons.

\subsubsection{Scalar leptoquark $(3,2,7 / 6)$}

The renormalizable LQ couplings for the state $\Delta(3,2,7 / 6)$ are [38]

$\mathcal{L}=\bar{\ell}_{R} Y_{L} \Delta^{\dagger} Q+\bar{u}_{R} Y_{R} \tilde{\Delta}^{\dagger} L+$ h.c.

The LQ Yukawa matrices $Y_{L}$ and $Y_{R}$ are written in the mass basis of up-type quarks and charged leptons with the CKM and PMNS rotations present in the down-type quarks and neutrinos. Thus, the couplings of LQ component with charge $5 / 3$ are

$\mathcal{L}^{(5 / 3)}=\left(\bar{\ell}_{R} Y_{L} u_{L}\right) \Delta^{(5 / 3) *}-\left(\bar{u}_{R} Y_{R} \ell_{L}\right) \Delta^{(5 / 3)}+$ h.c.

The tree-level amplitude induced by a nonchiral LQ state $\Delta^{(5 / 3)}$ involves both chiralities of fermions and is matched onto the set of (axial)vector, (pseudo)scalar, and (pseudo)tensor operators:

$$
\begin{aligned}
& C_{P}=C_{S}=-\frac{\pi}{2 \sqrt{2} G_{F} \alpha \lambda_{b}} \frac{Y_{\mu u}^{L *} Y_{c \mu}^{R *}}{m_{\Delta}^{2}}, \\
& -C_{P}^{\prime}=C_{S}^{\prime}=-\frac{\pi}{2 \sqrt{2} G_{F} \alpha \lambda_{b}} \frac{Y_{\mu c}^{L} Y_{u \mu}^{R}}{m_{\Delta}^{2}}, \\
& C_{T}=-\frac{\pi}{8 \sqrt{2} G_{F} \alpha \lambda_{b}} \frac{Y_{u \mu}^{R} Y_{\mu c}^{L}+Y_{c \mu}^{R *} Y_{\mu u}^{L *}}{m_{\Delta}^{2}}, \\
& C_{T 5}=-\frac{\pi}{8 \sqrt{2} G_{F} \alpha \lambda_{b}} \frac{-Y_{u \mu}^{R} Y_{\mu c}^{L}+Y_{c \mu}^{R *} Y_{\mu u}^{L *}}{m_{\Delta}^{2}}, \\
& C_{10}=C_{9}=\frac{\pi}{\sqrt{2} G_{F} \alpha \lambda_{b}} \frac{Y_{\mu c}^{L} Y_{\mu u}^{L *}}{m_{\Delta}^{2}} \\
& -C_{10}^{\prime}=C_{9}^{\prime}=\frac{\pi}{\sqrt{2} G_{F} \alpha \lambda_{b}} \frac{Y_{c \mu}^{R *} Y_{u \mu}^{R}}{m_{\Delta}^{2}} .
\end{aligned}
$$

In the minimal numerical scenario, strict bounds in the downtype quark sector can be evaded completely by putting to zero the couplings to the left-handed quarks. In this case we are allowed to have significant contributions to rare charm decays via the $C_{9}^{\prime}=-C_{10}^{\prime}$ contributions for which the bound from 
the last line of Table 2 applies. The contribution to the $D^{0}-\bar{D}^{0}$ mixing amplitude is matched onto the effective Hamiltonian $\mathcal{H}=C_{6}\left(\bar{u}_{R} \gamma^{\mu} c_{R}\right)\left(\bar{u}_{R} \gamma_{\mu} c_{R}\right)$ with the effective coefficient at scale $m_{\Delta}$

$C_{6}\left(m_{\Delta}\right)=-\frac{\left(Y_{c \mu}^{R *} Y_{u \mu}^{R}\right)^{2}}{64 \pi^{2} m_{\Delta}^{2}}=-\frac{\left(G_{F} \alpha\right)^{2}}{32 \pi^{4}} m_{\Delta}^{2}\left(\tilde{C}_{10}^{\prime}\right)^{2}$.

We have assumed that the leptoquark does not couple to electrons or tau leptons. Hadronic matrix element of the above operator in mixing is customarily expressed as $\left\langle\bar{D}^{0}\left|\left(\bar{u}_{R} \gamma_{\mu} c_{R}\right)\left(\bar{u}_{R} \gamma^{\mu} c_{R}\right)\right| D^{0}\right\rangle=\frac{2}{3} m_{D}^{2} f_{D}^{2} B$, where the bag parameter in the $\overline{\mathrm{MS}}$ scheme $B_{D}(3 \mathrm{GeV})=0.757(27)(4)$ has been computed on the lattice by the ETM Collaboration with $2+1+1$ dynamical fermions [39]. The SM part of the mixing amplitude is poorly known due to its nonperturbative nature and the only robust bound on the LQ couplings is obtained by requirement that the mixing frequency (in the absence of CP violation) has to be smaller than the world average $x=2\left|M_{12}\right| / \Gamma=\left(0.49_{-0.15}^{+0.14}\right) \%$ as quoted by the HFAG [24],

$\left|r C_{6}\left(m_{\Delta}\right)\right| \frac{2 m_{D} f_{D}^{2} B_{D}}{3 \Gamma_{D}}<x$,

where $r=0.76$ is a renormalization factor due to running of $C_{6}$ from scale $m_{\Delta}=1 \mathrm{TeV}$ down to $3 \mathrm{GeV}$ [3]. Finally, we find a bound on $C_{9}^{\prime}$ slightly stronger than but comparable to the one obtained from $D^{0} \rightarrow \mu^{+} \mu^{-}$:

$\left|C_{6}\left(m_{\Delta}\right)\right|<2.5 \times 10^{-13} \mathrm{GeV}^{-2} \Longrightarrow\left|\tilde{C}_{9}^{\prime}, \tilde{C}_{10}^{\prime}\right|<0.34$.

One can imagine an extension of this scenario which would include also scalar and tensor operators. Namely, we consider a numerically tuned example with $m_{\Delta}=1 \mathrm{TeV}$ and large $Y_{c \mu}^{R}=3$. The bound on $C_{10}^{\prime}$ from $D^{0} \rightarrow \mu^{+} \mu^{-}$would then impose the smallness of the coupling $Y_{u \mu}^{R}, Y_{u \mu}^{R}<0.007$. Bounds of similar strengths are expected from $D^{0}-\bar{D}^{0}$ mixing. Now one can introduce a nonzero coupling to the lefthanded quark doublet $Y_{\mu u}^{L}$ that would, together with large $Y_{c \mu}^{R}$ contribute to the Wilson coefficients $C_{S, P}$ and $C_{T, T 5}$. However, a very strong bound on $C_{S}$ now emerges from $D^{0} \rightarrow \mu^{+} \mu^{-}$and limits the left-handed coupling, $Y_{\mu u}^{L}<$ $1.2 \times 10^{-3}$. Thus we can realize

$$
\begin{aligned}
& -\tilde{C}_{10}^{\prime}=\tilde{C}_{9}^{\prime}=0.63, \\
& \quad 4 \tilde{C}_{T}=4 \tilde{C}_{T 5}=\tilde{C}_{P}=\tilde{C}_{S}=-0.049,
\end{aligned}
$$

together with small enough $Y_{\mu u}^{L}=1.2 \times 10^{-3}$ to comply with the constraints from $B, K$ physics and four fermion operator constraints [40].

\subsubsection{Vector leptoquark $(3,1,5 / 3)$}

The interactions of the vector LQ state $V^{(5 / 3)}(3,1,5 / 3)$ with the SM fermions are contained in a single term at the renormalizable level:

$\mathcal{L}=Y_{i j}\left(\bar{\ell}_{i} \gamma_{\mu} P_{R} u_{j}\right) V^{(5 / 3) \mu}+$ h.c.

Generation indices are denoted $i, j$. Integrating out $V^{(5 / 3)}$ results in the right-handed current operators:

$C_{9}^{\prime}=C_{10}^{\prime}=\frac{\pi}{\sqrt{2} G_{F} \lambda_{b} \alpha} \frac{Y_{\mu c} Y_{\mu u}^{*}}{m_{V}^{2}}$.

On the other hand, the same combination of couplings enters the $D^{0}-\bar{D}^{0}$ mixing. We employ the same type of Hamiltonian as in the preceding section, this time with the Wilson coefficient:

$C_{6}\left(m_{V}\right)=\frac{\left(Y_{\mu u} Y_{\mu c}^{*}\right)^{2}}{32 \pi^{2} m_{V}^{2}}=\frac{\left(G_{F} \alpha\right)^{2}}{16 \pi^{4}} m_{V}^{2}\left(\tilde{C}_{10}^{\prime}\right)^{2}$.

A consequence of the bound (27) is that the rare decay Wilson coefficients are limited:

$\left|\tilde{C}_{9}^{\prime}, \tilde{C}_{10}^{\prime}\right|<0.24$.

The above knowledge of $C_{9,10}^{\prime}$ implies that the branching ratio of $D \rightarrow \pi \mu^{+} \mu^{-}$in the high- $q^{2}$ bin is at most $1.4 \times$ $10^{-8}$, where the long-distance uncertainties have been taken into account. The effect is twice smaller than the existing experimental bound.

\subsection{Two Higgs doublet model type III}

In the Two Higgs doublet model of type III (THDM III) the neutral Higgses have flavor changing couplings to the fermions. The spectrum includes two neutral scalars, $h$ and $H$, one pseudoscalar, $A$, and two charged scalars, $H^{ \pm}$. In the scenario with MSSM-like scalar potential their masses and mixing angles are related [41],

$\tan \beta=\frac{v_{u}}{v_{d}}, \quad \tan 2 \alpha=\tan 2 \beta \frac{m_{A}^{2}+m_{Z}^{2}}{m_{A}^{2}-m_{Z}^{2}}$,
$m_{H^{ \pm}}^{2}=m_{A}^{2}+m_{W}^{2}, \quad m_{H}^{2}=m_{A}^{2}+m_{Z}^{2}-m_{h}^{2}$,

where $\beta, \tan \beta=v_{u} / v_{d}$, is the angle that diagonalizes the mass matrix of the charged states, $\alpha$ is the mixing angle of neutral scalars. The vacuum expectation values are normalized to the electroweak vacuum expectation value, $v / \sqrt{2}=$ $\sqrt{v_{u}^{2}+v_{d}^{2}}=174 \mathrm{GeV}$. The part of the interaction Lagrangian responsible for FCNCs in the up-type quarks and charged leptons is [41] 


$$
\begin{aligned}
& \mathcal{L}=\frac{y_{i j}^{(\ell) H_{k}}}{\sqrt{2}} H_{k} \bar{\ell}_{L, i} \ell_{R, j}+\frac{y_{i j}^{(u) H_{k}}}{\sqrt{2}} H_{k} \bar{u}_{L, i} u_{R, j}+\text { h.c. }, \\
& H_{k}=(H, h, A),
\end{aligned}
$$

and the neutral Yukawa couplings for the charged leptons and up-type quarks are

$$
\begin{aligned}
y_{i j}^{(\ell) H_{k}} & =x_{d}^{k} \frac{m_{\ell_{i}}}{v_{d}} \delta_{i j}-\epsilon_{i j}^{\ell}\left(x_{d}^{k} \tan \beta-x_{u}^{k *}\right), \\
y_{i j}^{(u) H_{k}} & =x_{u}^{k} \frac{m_{u_{i}}}{v_{u}} \delta_{i j}-\epsilon_{i j}^{u}\left(x_{u}^{k} \cot \beta-x_{d}^{k *}\right),
\end{aligned}
$$

respectively. The flavor off-diagonal terms $\epsilon_{f i}^{\ell}, \epsilon_{f i}^{u}$ are free parameters of the model. The coefficients $x_{q}^{k}$ for $H_{k}=$ $(H, h, A)$ are determined by the mixing angles of the neutral scalars and the VEVs [41]

$x_{u}^{k}=(-\sin \alpha,-\cos \alpha, i \cos \beta)$,

$x_{d}^{k}=(-\cos \alpha, \sin \alpha, i \sin \beta)$.

For the transitions $c \rightarrow u \ell^{+} \ell^{-}$the driving flavor changing parameter is $\epsilon_{12}^{u}$, which induces scalar and pseudoscalar Wilson coefficients, while we assume that $\epsilon_{22}^{\ell}$ is negligible [41]:

$$
\begin{aligned}
& -C_{P}=C_{S}=\frac{\pi}{4 \sqrt{2} G_{F} \alpha \lambda_{b}} \frac{m_{\mu}}{v} \frac{\epsilon_{12}^{u *} \tan \beta}{m_{H}^{2}}, \\
& C_{P}^{\prime}=C_{S}^{\prime}=\frac{\pi}{4 \sqrt{2} G_{F} \alpha \lambda_{b}} \frac{m_{\mu}}{v} \frac{\epsilon_{21}^{u} \tan \beta}{m_{H}^{2}} .
\end{aligned}
$$

The best upper bounds on $C_{P}, C_{S}$, or $C_{P}^{\prime}, C_{S}^{\prime}$ pairs are obtained from $\operatorname{BR}\left(D^{0} \rightarrow \mu^{+} \mu^{-}\right)$and read $\left|\tilde{C}_{S}-\tilde{C}_{S}^{\prime}\right| \leq 0.05$ and $\left|\tilde{C}_{P}-\tilde{C}_{P}^{\prime}\right| \leq 0.05$, which makes them very difficult to probe in $D \rightarrow \pi \mu^{+} \mu^{-}$decay, unless the cancellation between $C_{S}\left(C_{P}\right)$ and $C_{S}^{\prime}\left(C_{P}^{\prime}\right)$ in $D^{0} \rightarrow \mu^{+} \mu^{-}$is arranged by fine-tuning.

\subsection{Flavor specific $Z^{\prime}$ extension}

An additional neutral gauge boson appears in many extensions of the SM. Current searches for $Z^{\prime}$ at the LHC are well motivated by many extensions of the SM; see e.g. [42,43]. Even more, a $Z^{\prime}$ boson can explain the $B \rightarrow K^{*} \mu^{+} \mu^{-}$ angular asymmetries puzzle, as presented in e.g. [44,45]. Assuming as in [43] flavor nonuniversal couplings of $Z^{\prime}$ to fermions, we allow $Z^{\prime}$ to couple only to the pair $\bar{c} u$ and $c \bar{u}$. Such a model in the most general way has been considered by the authors of [3]. In order to avoid constraints coming from the down-type quark sector which will affect left-handed quark couplings, we allow only right-handed couplings of $\mathcal{L}_{Z^{\prime}}^{q}=C^{u}\left(\bar{u} \gamma^{\mu} P_{R} c\right) Z_{\mu}^{\prime}$. This assumption leads to the same effective operator $\mathcal{H}^{\text {eff }}=C_{6}\left(\bar{u} \gamma_{\mu} P_{R} c\right)\left(\bar{u} \gamma^{\mu} P_{R} c\right)$ as already discussed in the case of leptoquarks. The effective Wilson coefficient describing the $D^{0}-\bar{D}^{0}$ transition is now
$C_{6}\left(m_{Z^{\prime}}\right)=\frac{\left|C^{u}\right|^{2}}{2 m_{Z^{\prime}}^{2}}$.

The bound on $C_{6}$ (27) leads to $\left|C^{u}\right|<7.1 \times 10^{-4}\left(m_{Z^{\prime}} \mid\right.$ $1 \mathrm{TeV})$. Allowing $Z^{\prime}$ to couple to muons as in the SM with $g_{L}^{\ell}=\left(g / \cos \theta_{W}\right)\left(-1 / 2+\sin ^{2} \theta_{W}\right)$ and $g_{R}^{\ell}=$ $g \sin ^{2} \theta_{W} / \cos \theta_{W}$, we obtain

$C_{9}^{\prime}=\frac{4 \pi}{\sqrt{2} G_{F} \lambda_{b} \alpha} \frac{\left(g_{L}^{\ell}+g_{R}^{\ell}\right) C^{u}}{2 m_{Z^{\prime}}^{2}}$

and

$C_{10}^{\prime}=\frac{4 \pi}{\sqrt{2} G_{F} \lambda_{b} \alpha} \frac{\left(-g_{L}^{\ell}+g_{R}^{\ell}\right) C^{u}}{2 m_{Z^{\prime}}^{2}}$.

For $m_{Z^{\prime}} \sim 1 \mathrm{TeV}$ this amounts to $\left|C_{9}\right| \lesssim 8$ and $\left|C_{10}\right| \lesssim 100$ $\left(\left|\tilde{C}_{9}\right|<10^{-3}\right.$, and $\left.\left|\tilde{C}_{10}\right|<0.014\right)$; this induces negligible effects in $D \rightarrow \pi \mu^{+} \mu^{-}$and $D \rightarrow \mu^{+} \mu^{-}$decays.

\section{Lepton flavor universality violation}

Lepton flavor universality was checked in the case of $B \rightarrow$ $K \ell^{+} \ell^{-}$with $\ell=e, \mu$ by the LHCb experiment [15] in the low dilepton invariant mass region, $q^{2} \in[1,6] \mathrm{GeV}^{2}$. The disagreement between the measurement and the value predicted within the $\mathrm{SM}$ is $2.6 \sigma$ [46]. This disagreement might be a result of NP, as first pointed out in Ref. [46]. Many subsequent studies found a number of models which can account for the observed discrepancy. In the following we assume that the amplitude for $D^{+} \rightarrow \pi^{+} e^{+} e^{-}$receives SM contributions only, while in the case of the $\pi^{+} \mu^{+} \mu^{-}$mode, there can be NP contributions, similarly to what was assumed for $R_{K}$ in Ref. [47]. We define LFU ratios in the low- and high- $q^{2}$ regions by

$R_{\pi}^{\mathrm{I}}=\frac{\mathrm{BR}\left(D^{+} \rightarrow \pi^{+} \mu^{+} \mu^{-}\right) q^{2} \in\left[0.25^{2}, 0.525^{2}\right] \mathrm{GeV}^{2}}{\mathrm{BR}\left(D^{+} \rightarrow \pi^{+} e^{+} e^{-}\right) q^{2} \in\left[0.25^{2}, 0.525^{2}\right] \mathrm{GeV}^{2}}$

and

$R_{\pi}^{\mathrm{II}}=\frac{\operatorname{BR}\left(D^{+} \rightarrow \pi^{+} \mu^{+} \mu^{-}\right)_{q^{2} \in\left[1.25^{2}, 1.73^{2}\right] \mathrm{GeV}^{2}}}{\operatorname{BR}\left(D^{+} \rightarrow \pi^{+} e^{+} e^{-}\right)_{q^{2} \in\left[1.25^{2}, 1.73^{2}\right] \mathrm{GeV}^{2}}}$

In the SM the departure of the above ratios from 1 comes entirely from the lepton mass differences. We find $R_{\pi}^{\mathrm{I}, \mathrm{SM}}=$ $0.87 \pm 0.09$ in the low- $q^{2}$ and $R_{\pi}^{\mathrm{II}, \mathrm{SM}}=0.999 \pm 0.001$ in the high- $q^{2}$ region, where in the latter region both leptons are effectively massless. In Table 3 we quote ranges for the ratio $R_{\pi}^{\mathrm{II}}$ for the maximal allowed values of Wilson coefficients by rare charm decays considered in the previous sections. Generally we find that with currently allowed Wilson coefficients and assuming no NP contribution in the electronic modes these ratios could become much larger. The spread in these predictions is large because of the unknown relative phases in the resonant part of the spectrum, i.e., $\operatorname{BR}\left(D^{+} \rightarrow\right.$ 
Table 3 The LFU ratio $R_{\pi}^{\mathrm{II}}$ at high dilepton invariant mass bin and maximal value of each Wilson coefficient (applies also for the primed coefficients, $\tilde{C}_{i}^{\prime}$ ). It is assumed that NP contributes only to the muonic mode. The $\mathrm{SM}$ value of $R_{\pi}^{\mathrm{II}}$ is given in the first row

\begin{tabular}{lll}
\hline & $\left|\tilde{C}_{i}\right|_{\max }$ & $R_{\pi}^{\mathrm{II}}$ \\
\hline $\mathrm{SM}$ & - & $0.999 \pm 0.001$ \\
$\tilde{C}_{7}$ & 1.6 & $\sim 6-100$ \\
$\tilde{C}_{9}$ & 1.3 & $\sim 6-120$ \\
$\tilde{C}_{10}$ & 0.63 & $\sim 3-30$ \\
$\tilde{C}_{S}$ & 0.05 & $\sim 1-2$ \\
$\tilde{C}_{P}$ & 0.05 & $\sim 1-2$ \\
$\tilde{C}_{T}$ & 0.76 & $\sim 6-70$ \\
$\tilde{C}_{T 5}$ & 0.74 & $\sim 6-60$ \\
$\tilde{C}_{9}= \pm \tilde{C}_{10}$ & 0.63 & $\sim 3-60$ \\
$\tilde{C}_{9}^{\prime}=-\left.\tilde{C}_{10}^{\prime}\right|_{\mathrm{LQ}(3,2,7 / 6)}$ & 0.34 & $\sim 1-20$ \\
\hline
\end{tabular}

$\left.\pi^{+} e^{+} e^{-}\right) \approx \operatorname{BR}\left(D^{+} \rightarrow \pi^{+} \mu^{+} \mu^{-}\right) \approx(0.5-5.3) \times 10^{-9}$. Note that large enhancements are allowed in the scenarios which are currently constrained by $D^{+} \rightarrow \pi^{+} \mu^{+} \mu^{-}$. In the low- $q^{2}$ region the interference terms in $R_{\pi}^{\mathrm{I}}$ are even more pronounced since the effect of nearby $\rho$ resonance is interfering either in positive or in negative direction, and thus we cannot conclude the sign of the deviation from the SM value of $R_{\pi}^{\mathrm{I}}$.

\section{Summary and outlook}

Motivated by the great improvement of the bounds on rare charm decays by the LHCb experiment we determine the bounds on the effective Wilson coefficients. Existing data implies upper bounds on the effective Wilson coefficients as presented in Table 2. The strongest constraints on $C_{10}$, $C_{P}, C_{S}$, and $C_{10}^{\prime}, C_{P}^{\prime}, C_{S}^{\prime}$ are obtained from the bound on the branching fraction of $D^{0} \rightarrow \mu^{+} \mu^{-}$decay. The nonresonant differential decay width distribution gives bounds on $C_{i}$, $i=7,9,10, S, P, T, T 5$ as well as on the coefficients of the operators of opposite chirality. The constraints are stricter in the high dilepton invariant mass bin than in the low dilepton invariant mass bin, and this statement applies in particular to the contributions of the scalar and pseudoscalar operators. The forward-backward asymmetry is sensitive to the combination of scalar and tensor coefficients at high- $q^{2}$.

Next, we have investigated new physics models in which the effective operators may be generated. We have found that the presence of a leptoquark, which is either a scalar and weak doublet, $(3,2,7 / 6)$, or has spin- 1 and is a weak singlet, $(3,1,5 / 3)$, can lead to sizable contributions to the Wilson coefficients $C_{9}^{\prime}$ and $C_{10}^{\prime}$. The sensitivity to the LQ scenarios is similar in the high- $q^{2}$ bin of $D^{+} \rightarrow \pi^{+} \mu^{+} \mu^{-}$and $D^{0} \rightarrow$ $\mu^{+} \mu^{-}$, while $D^{0}-\bar{D}^{0}$ mixing results in a somewhat stronger constraint. For the Two Higgs doublet model of type III the presence of scalar and pseudoscalar operators enhances the sensitivity in $D^{0} \rightarrow \mu^{+} \mu^{-}$and therefore results in small effects in $D^{+} \rightarrow \pi^{+} \mu^{+} \mu^{-}$. We have also discussed a SM extension by a $Z^{\prime}$ gauge boson where the tree-level amplitude in $D^{0}-\bar{D}^{0}$ mixing is a dominant constraint and leaves no possibility of signals in rare charm decays.

Our study indicates the possibility to check whether lepton flavor universality between muonic and electronic channels is valid by means of studying ratios of widths of $D^{+} \rightarrow$ $\pi^{+} \ell^{+} \ell^{-}$at low or high dilepton invariant mass bins, $R_{\pi}^{\mathrm{I}, \mathrm{II}}$. In the SM the two ratios are close to 1, especially in the high$q^{2}$ bin. Assuming the electronic decay is purely SM-like, we find that in the high- $q^{2}$ bin the ratio $R_{\pi}^{\mathrm{II}}$ is in most cases significantly increased with respect to the SM prediction, while there is no clear preference between higher and lower values at low- $q^{2}$ bin ratio $R_{\pi}^{\mathrm{I}}$. In the leptoquark models studied in this paper the ratio may be greatly increased, but a slight decrease cannot be excluded, presently due to the unknown interplay of weak phases with the phases of the resonant spectrum. Chances to observe new physics in rare charm decays are present in models where the connection to the stringent constraints stemming from $B$ and $K$ flavor physics are hindered. New physics models which fulfill this condition are main candidates to be exposed experimentally by future progress in bounding the rare charm decays $D \rightarrow \pi \mu^{+} \mu^{-}$ and $D^{0} \rightarrow \mu^{+} \mu^{-}$, as well as by more precise studies of $D^{0}-\bar{D}^{0}$ mixing with the potential NP contributions. Alternatively, experimental tests of lepton flavor universality in rare charm decays might point toward the presence of new physics in the charm sector, which can easily be hidden in the case of existing experimental observables.

Note While we were finishing this paper another work [48] appeared in which the authors studied rare charm decays.

Acknowledgments We thank Benoit Viaud for constructive comments on the first version of this paper. We thank Martin Bauer for pointing out a typographical error in Eq. (16) in the previous version. We acknowledge support of the Slovenian Research Agency. This research was supported in part by the Munich Institute for Astro- and Particle Physics (MIAPP) of the DFG cluster of excellence "Origin and Structure of the Universe".

Open Access This article is distributed under the terms of the Creative Commons Attribution 4.0 International License (http://creativecomm ons.org/licenses/by/4.0/), which permits unrestricted use, distribution, and reproduction in any medium, provided you give appropriate credit to the original author(s) and the source, provide a link to the Creative Commons license, and indicate if changes were made.

Funded by SCOAP $^{3}$.

\section{References}

1. S.L. Glashow, J. Iliopoulos, L. Maiani, Phys. Rev. D 2, 1285 (1970). doi:10.1103/PhysRevD.2.1285

2. G. Isidori, Y. Nir, G. Perez, Ann. Rev. Nucl. Part. Sci. 60, 355 (2010). doi:10.1146/annurev.nucl.012809.104534 
3. E. Golowich, J. Hewett, S. Pakvasa, A.A. Petrov, Phys. Rev. D 79, 114030 (2009). doi:10.1103/PhysRevD.79.114030

4. C. Greub, T. Hurth, M. Misiak, D. Wyler, Phys. Lett. B 382, 415 (1996). doi:10.1016/0370-2693(96)00694-6

5. G. Burdman, E. Golowich, J.L. Hewett, S. Pakvasa, Phys. Rev. D 52, 6383 (1995). doi:10.1103/PhysRevD.52.6383

6. S. De Boer, Talk given at Charm 2015 Conference, Detroit, 17-22 May 2015 (2015)

7. S. Fajfer, P. Singer, J. Zupan, Eur. Phys. J. C 27, 201 (2003). doi:10. 1140/epjc/s2002-01090-5

8. Q. Ho-Kim, X.Y. Pham, Phys. Rev. D 61, 013008 (2000). doi:10. 1103/PhysRevD.61.013008

9. G. Burdman, E. Golowich, J.L. Hewett, S. Pakvasa, Phys. Rev. D 66, 014009 (2002). doi:10.1103/PhysRevD.66.014009

10. I. Dorsner, S. Fajfer, J.F. Kamenik, N. Kosnik, Phys. Lett. B 682, 67 (2009). doi:10.1016/j.physletb.2009.10.087

11. A. Greljo, G. Isidori, D. Marzocca, JHEP 07, 142 (2015). doi:10. 1007/JHEP07(2015)142

12. A.J. Buras, D. Buttazzo, J. Girrbach-Noe, R. Knegjens, JHEP 11, 121 (2014). doi:10.1007/JHEP11(2014)121

13. R. Aaij et al., Phys. Lett. B 725, 15 (2013). doi:10.1016/j.physletb. 2013.06.037

14. R. Aaij et al., Phys. Lett. B 724, 203 (2013). doi:10.1016/j.physletb. 2013.06.010

15. R. Aaij et al., Phys. Rev. Lett. 113, 151601 (2014). doi:10.1103/ PhysRevLett.113.151601

16. G. Hiller, F. Kruger, Phys. Rev. D 69, 074020 (2004). doi:10.1103/ PhysRevD.69.074020

17. G. Isidori, J.F. Kamenik, Z. Ligeti, G. Perez, Phys. Lett. B 711, 46 (2012). doi:10.1016/j.physletb.2012.03.046

18. S. Fajfer, S. Prelovsek, Phys. Rev. D 73, 054026 (2006). doi:10. 1103/PhysRevD.73.054026

19. D. Becirevic, A.B. Kaidalov, Phys. Lett. B 478, 417 (2000). doi:10. 1016/S0370-2693(00)00290-2

20. D. Besson et al., Phys. Rev. D 80, 032005 (2009). doi:10.1103/ PhysRevD.80.032005

21. S. Dobbs et al., Phys. Rev. D 77, 112005 (2008). doi:10.1103/ PhysRevD.77.112005

22. M. Ablikim et al., BESIII Collaboration, Phys. Rev. D 92, 072012 (2015)

23. J.P. Lees et al., Phys. Rev. D 91(5), 052022 (2015). doi:10.1103/ PhysRevD.91.052022

24. Y. Amhis et al., Heavy Flavor Averaging Group. arXiv:1412.7515 (2014)

25. H. Na, C.T.H. Davies, E. Follana, J. Koponen, G.P. Lepage, J. Shigemitsu, Phys. Rev. D 84, 114505 (2011). doi:10.1103/ PhysRevD.84.114505

26. S. Fajfer, N. Košnik, Phys. Rev. D 87(5), 054026 (2013). doi:10. 1103/PhysRevD.87.054026
27. C. Bobeth, G. Hiller, G. Piranishvili, JHEP 12, 040 (2007). doi:10. 1088/1126-6708/2007/12/040

28. S. Fajfer, N. Kosnik, S. Prelovsek, Phys. Rev. D 76, 074010 (2007). doi:10.1103/PhysRevD.76.074010

29. S. Prelovsek, Weak decays of heavy mesons. Ph.D. thesis, Ljubljana University, Ljubljana (2000)

30. S. Fajfer, S. Prelovsek, P. Singer, Phys. Rev. D 64, 114009 (2001). doi:10.1103/PhysRevD.64.114009

31. K.A. Olive et al., Chin. Phys. C 38, 090001 (2014). doi:10.1088/ 1674-1137/38/9/090001

32. H. Na, C.T.H. Davies, E. Follana, G.P. Lepage, J. Shigemitsu, Phys. Rev. D 86, 054510 (2012). doi:10.1103/PhysRevD.86.054510

33. A. Bazavov et al., Phys. Rev. D 85, 114506 (2012). doi:10.1103/ PhysRevD.85.114506

34. S. Aoki, Eur. Phys. J. C 74, 2890 (2014). doi:10.1140/epjc/ s10052-014-2890-7 (January 6 et al., update, 2015)

35. J.P. Lees et al., Phys. Rev. D 85, 091107 (2012). doi:10.1103/ PhysRevD.85.091107

36. D. Becirevic, N. Kosnik, F. Mescia, E. Schneider, Phys. Rev. D 86 , 034034 (2012). doi:10.1103/PhysRevD.86.034034

37. S. Fajfer, N. Kosnik, Phys. Rev. D 79, 017502 (2009). doi:10.1103/ PhysRevD.79.017502

38. I. Doršner, S. Fajfer, N. Košnik, I. Nišandžić, JHEP 11, 084 (2013). doi:10.1007/JHEP11(2013)084

39. N. Carrasco, P. Dimopoulos, R. Frezzotti, V. Lubicz, G.C. Rossi, S Simula, C. Tarantino, Phys. Rev. D 92(3), 034516 (2015). doi:10. 1103/PhysRevD.92.034516

40. M. Carpentier, S. Davidson, Eur. Phys. J. C 70, 1071 (2010). doi:10. 1140/epjc/s10052-010-1482-4

41. A. Crivellin, A. Kokulu, C. Greub, Phys. Rev. D 87(9), 094031 (2013). doi:10.1103/PhysRevD.87.094031

42. A. Celis, J. Fuentes-Martin, M. Jung, H. Serodio, Phys. Rev. D 92(1), 015007 (2015). doi:10.1103/PhysRevD.92.015007

43. P. Langacker, M. Plumacher, Phys. Rev. D 62, 013006 (2000). doi:10.1103/PhysRevD.62.013006

44. S. Descotes-Genon, L. Hofer, J. Matias, J. Virto, J. Phys. Conf. Ser. 631(1), 012027 (2015). doi:10.1088/1742-6596/631/1/012027

45. A.J. Buras, F. De Fazio, J. Girrbach, JHEP 02, 116 (2013). doi:10. 1007/JHEP02(2013)116

46. G. Hiller, M. Schmaltz, Phys. Rev. D 90, 054014 (2014). doi:10. 1103/PhysRevD.90.054014

47. D. Bečirević, S. Fajfer, N. Košnik, Phys. Rev. D 92(1), 014016 (2015). doi:10.1103/PhysRevD.92.014016

48. S. de Boer, G. Hiller. arXiv:1412.7515 (2015) 